\title{
Prevalence and Patterns of Thyroid Dysfunction in Patients with Rheumatoid Arthritis
}

\author{
Hala H. Mosli ${ }^{*}$ and Suzan M. Attar
}

Department of Medicine, King Abdulaziz University, Jeddah, Kingdom of Saudi Arabia

\begin{abstract}
Background: The relationship between Rheumatoid Arthritis (RA) and Autoimmune Thyroid disturbance is one of the numerous examples of autoimmune comorbidities that are already established. This has been investigated in many countries and different populations, but data are still missing in some countries. We aimed to investigate comorbid RA and autoimmune thyroid disturbance in the Saudi population.

Methods: We conducted this retrospective study at a tertiary-teaching center, in Saudi Arabia. We reviewed data of all RA patients, over the last 14 years, and selected all those who had thyroid function results recorded in their laboratory data (free thyroxine, free triiodothyronine and Thyroid-stimulating hormone). We examined all laboratory results of the sample, to assess prevalence of different thyroid disturbances, in regards to the laboratory standard results. We also analyzed the relationship between abnormal thyroid results and the usual inflammatory and autoimmune parameters: Erythrocyte Sedimentation Rate (ESR), C-Reactive Protein level (CRP), Rheumatoid Factor (RF) and AntiCycliccitrullinated Peptide (anti-CCP). We used both Chi-square testing and Pearson correlation test, whenever indicated, and $P$-value less than 0.05 to assess significance.

Results: One hundred and fifty one (151) patients had their thyroid function tests recorded. We observed the following: high prevalence of thyroid function disturbances in our sample of RA patients, compared to non-RA patients]. (26.3\% and $7 \%$ respectively), with the patterns of thyroid dysfunction as follows: Subclinical hypothyroidism in 29 patients (19\%), Hypothyroidism in 6 patients (4\%), Subclinical hyperthyroidism in 4 patients $(2.6 \%)$ and hyperthyroidism in 1 patient $(0.7 \%)$. No patients were recorded as Euthyroid sick syndrome. Also there was a significant positive correlation between TSH and CRP (r=0.22, $P=0.029)$.

Conclusion: There is a high prevalence of comorbid hypothyroidism in our RA patients, in a tertiary care hospital in Western Saudi Arabia. Physicians should be aware of screening RA patients for this comorbidity, especially when the specific symptoms may overlap between both diagnoses.
\end{abstract}

Keywords: Autoimmune thyroid disease, prevalence, rheumatoid arthritis, Saudi Arabia, screening, subacute thyroiditis, thyroid.

\section{INTRODUCTION}

The link between rheumatoid arthritis (RA) and autoimmune thyroid disease (AITD) has been an area of interest as early as the 1960's [1]. This presumed association has been researched in various parts of the world, with mixed results; some studies found an association does indeed exist, while other papers disputed this relationship [2], including some parts of the Middle East, such as Egypt and Kuwait [1, 3-6]. In Saudi Arabia, there is little epidemiological data about rheumatoid arthritis, in general, and the only study performed in 1998, by Al-Dalaan et al, describes the prevalence of RA in the region of Al-Qassim, estimating it to 2.2 per thousand people [7]. However, due the limited area where this study was conducted, as well as the diverse environments and ethnic backgrounds of people in the different regions of this large country, this data cannot

*Address correspondence to this author at the Department of Medicine, King Abdulaziz University, P.O. Box 80215, Jeddah 21589, Kingdom of Saudi Arabia; Tel: +966-2-6408243; Fax: +966-2-6408315;

E-mail: halamosli100@gmail.com be representative of the whole population of Saudi Arabia. Similarly, data regarding the prevalence of autoimmune thyroid disease is also scarce in Saudi Arabia. A study by Akbar et al. (2006) estimated thyroid dysfunction at $7 \%$ in healthy, non-diabetics as compared to patients with type 2 diabetes [8].

Given the autoimmune nature of both diseases and the reported data from the other areas of the Middle East, some Saudi physicians may expect the coexistence of AITD with RA and do appropriately screen their patients. However, availability of national data on the prevalence of that comorbidity would be very useful for the Saudi physicians, as well as for authorities, to determine a standard level of clinical awareness or to further investigate reliability and cost-effectiveness of systematic screening.

The objective of our study was to determine the prevalence of the coexistence of thyroid dysfunction with rheumatoid arthritis in Saudi Arabia, in a cohort of 151 RA patients presenting to the Rheumatology Clinic at a teaching hospital in Jeddah, in western region of Saudi Arabia. 


\section{MATERIALS AND METHODS}

This retrospective study was conducted in 2013, at King Abdulaziz University Hospital (KAUH), a teaching hospital in Jeddah. The study was approved by the Biomedical Ethical Research Committee of the Faculty of Medicine of King Abdulaziz University (KAU).

We reviewed the files of all RA patients diagnosed and/or followed up) in the hospital, from 2000 to 2013. RA patients were diagnosed using the $9^{\text {th }}$ edition of the International Classification of Diseases (ICD-9), codes for RA (714.0-714.33); but we reevaluated the files to include only patients who fulfill the $2010 \mathrm{ACR} /$ European League Against Rheumatism (EULAR) classification criteria for rheumatoid arthritis [9]. The other inclusion criterion was the availability in the patient's record of thyroid function test results.

The following data were collected from the patients' files: demographic features, duration of the disease at the time of the study (in years -yrs), history of thyroid disease, and use of thyroid replacement. The following investigations were collected from the patients clinical records: Erythrocyte sedimentation rate (ESR: normal $0-20 \mathrm{~mm} / \mathrm{hr}$ ), C-reactive protein level (CRP: reference range 0-3 mg/L ), Rheumatoid factor (RF: normal 0-20 IU/L measured by nephelometry), Anti-Cycliccitrullinated peptide (Anti-CCP: normal 0-20 EU measured by ELISA), Thyroid function test (TFT including: Free thyroxine $\left(\mathrm{FT}_{4}\right.$ : normal 9.2-23.8 $\mathrm{pg} \mathrm{mL} \mathrm{m}^{-1}$ ), Free triiodothyronine $\left(\mathrm{FT}_{3:}\right.$ normal 1.9-4.8 $\left.\mathrm{pg} \mathrm{mL}^{-1}\right)$ and Thyroidstimulating hormone (TSH: normal $0.23-4.0 \mathrm{mU} / \mathrm{L}$ ) were measured by immunochemiluminescent assay on the IMMULITE 1000 (Diagnostic Products Corporation, Los Angeles, Calif., USA).

Thyroid disturbances were further classified into: Euthyroid: If $\mathrm{FT}_{4}, \mathrm{FT}_{3}$ and $\mathrm{TSH}$ were normal; Hypothyroidism: if the TSH was raised together with a decreased $\mathrm{FT}_{4}$; Hyperthyroidism: if the TSH was low (TSH $<0.20 \mathrm{mU} / \mathrm{L}$ ) with normal or high $\mathrm{FT}_{4}$; Subclinical hypothyroidism if the TSH was high level with normal $\mathrm{FT}_{4}$; Subclinical hyperthyroidism: if TSH was low with normal $\mathrm{FT}_{4}$; Euthyroid sick syndrome: if TSH was normal with Normal/low FT $[10,11]$.

\section{Statistical Analysis}

Data analysis was done using Statistical Package for Social Sciences (SPSS software version-20). Means and standard deviations were calculated for quantitative data and proportions for categorical variables. To establish the relationship between categorical variables, Chi square testing was used and Independent t-test was used between continues variables with normal distribution assumed. Furthermore, regardless of categories, Pearson correlation Test was used to check the dependency of two continues variables. A $P$-value $<0.05$ is considered in rejecting null hypothesis.

\section{RESULTS}

One hundred and fifty one (151) RA patients were available with TFT done.
119 patients $(88 \%)$ were women. The mean $( \pm \mathrm{SD})$ age at the time of the study was $46.96( \pm 12.5)$ years for females and $44.6( \pm 13.2)$ for males. Sixty-three $(41.7 \%)$ of our patients were Saudi. The mean (SD) duration of the disease, at the time of the study, was 6.14 years $( \pm 6)$. Only 24 patients $(16 \%)$ were receiving thyroid treatment. The mean ESR was $27.48 \mathrm{~mm} / \mathrm{hr}(+27.48)$, and mean CRP $12.9(+12.9) \mathrm{mg} / \mathrm{L}$. RF was positive in 84 patients $(56 \%)$, while the anti-CCP was positive in 77 patients $(51 \%)$.

The following TFT abnormalities were detected: Subclinical hypothyroidism: in 29 patients (19.2\%), Hypothyroidism: in 6 patients (4\%), Subclinical hyperthyroidism: in 4 patients $(2.6 \%)$ and the least was Hyperthyroidism: in 1 patient $(0.7 \%)$. No patients were recorded as Euthyroid sick syndrome. Table 1 shows the frequency of thyroid disturbance in RA patients.

Table 1. Frequency of thyroid disturbance in 151 RA patients.

\begin{tabular}{|c|c|c|}
\hline Variables & Number & Percent \\
\hline \hline Euothyroidism & 110 & 73 \\
\hline Clinical-Hypothyroidism & 6 & 4 \\
\hline Clinical-Hyperthyrodism & 1 & 0.7 \\
\hline Subclinical-Hypothyroidism & 29 & 19.2 \\
\hline Subclinical-Hyperthyrodism & 4 & 2.6 \\
\hline Sickeuothyroid disease & 0 & 0 \\
\hline
\end{tabular}

By Chi-square testing there was a significant association between clinical hypothyroidism and $\mathrm{RF}(P=0.027)$. Table 2 demonstrates the association between the thyroid status and $\mathrm{RF}$, anti-CCP and CRP.

By Pearson correlation, there was a significant positive correlation between TSH and CRP $(\mathrm{r}=0.22, P=0.029)$, which means the higher the CRP, the higher TSH and the more the patient is likely to have either clinical hypothyroidism or subclinical hypothyroidism.

\section{DISCUSSION}

We conducted this retrospective cross-sectional study to assess the co-existence of thyroid dysfunction in a cohort of RA patients presenting to the Rheumatology Outpatient Department at a University Teaching Hospital. All patients had proven RA as described in the Methods section, and data was collected from their files regarding thyroid status.

Data regarding thyroid disease in Saudi Arabia is scarce, with very little information detailing the patterns of thyroid dysfunction in the Kingdom. One study by Akbar et al. in 2006 [8] found that thyroid autoimmunity existed in 5\% of non-diabetic individuals, while thyroid dysfunction from any etiology was diagnosed in $7 \%$ of non-diabetics.

Autoimmune disease tends to affect females more than males, with varying frequency according to disease and organ/body system. Ngo et al. found that rheumatoid arthritis 
Table 2. Association between Different variables with the thyroid disturbance status in 151 RA patients.

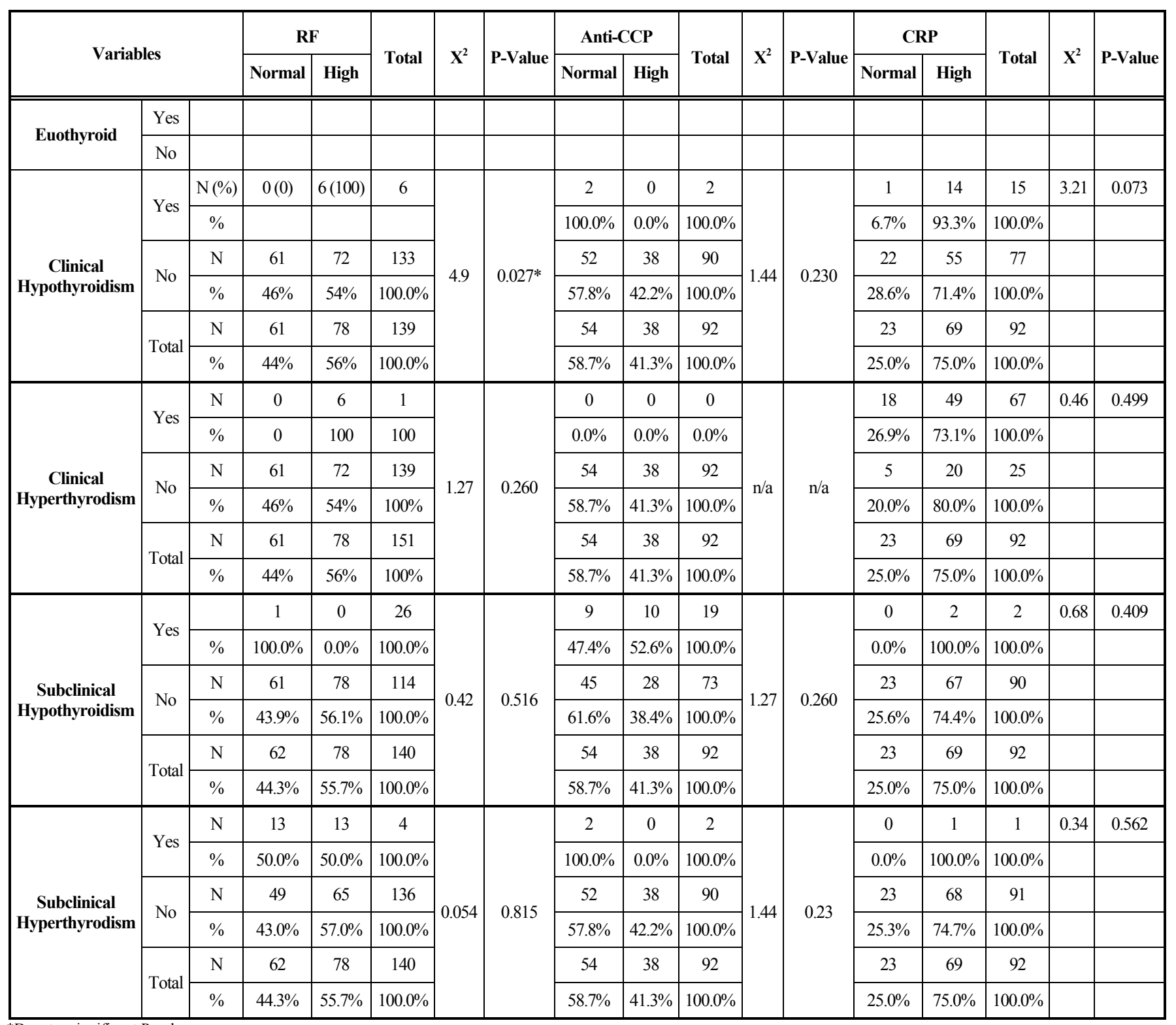

*Denotes significant P-value.

was more frequent in females than males, with a $66-86 \%$ higher rate in females. Autoimmune thyroid disease in all its forms was also found to be more frequent in females than males in this paper [10].

There is a high frequency of comorbid thyroid dysfunction in RA, since $27 \%$ of patients in our cohort have significant abnormal thyroid results in their laboratory records. The most frequent thyroid dysfunction found was subclinical hypothyroidism with $19.2 \%$ of the patients.

The definition of hypothyroidism based on TSH values is under continued debate. In our study, we have considered 4.1 $\mathrm{IU} / \mathrm{mL}$ as an upper limit $[11,12]$, a TSH range of 4.1-10 $\mathrm{IU} / \mathrm{mL}$ plus symptoms to diagnose subclinical hypothyroidism and a TSH above $10 \mathrm{IU} / \mathrm{mL}$ to diagnose clinical hypothyroidism.
Thyroid dysfunction of autoimmune etiology has long been linked to other autoimmune diseases. El-Sherif et al. published data demonstrating that in a cohort of Egyptian patients with systemic lupus erythematosus (SLE) and rheumatoid arthritis (RA), thyroid dysfunction was found in $50 \%$ of SLE patients and $15 \%$ of RA patients [4].

Several genes were established to carry an increased risk of developing autoimmune thyroid disease, including CD40, CTLA4, PTPN22, TSH receptor gene, thyroglobulin gene and HLA gene complex [2,13-15]. Some of these genes, such as CD40, CTLA4, PTPN22, and also, the HLA gene complex play a role in the development of rheumatoid arthritis [14]. This overlap may explain the co-existence of these conditions.

While many causes for hypothyroidism exist, the most common cause, especially in older women, remains autoimmune thyroiditis (Hashimoto's thyroiditis) [16]. This 
population of patients is also presumed to be at risk for developing other autoimmune diseases such as RA.

The common autoimmune etiology for both RA and AITD makes their co-existence sound logical and even somehow expected, but the exact mechanism by which they coexist remains unclear. Many theories have been proposed to explain this co-existence, one of which suggested that auto-reactive cells responsible for the underlying pathology in RA may also provoke an autoimmune thyroiditis causing Hashimoto's thyroiditis [3]. Also, environmental factors may precipitate these events $[17,18]$.

In our study, further analysis of presence of RA markers and markers of inflammation revealed a statistically significant association between RF-positive RA and clinical hypothyroidism, where $4.9 \%$ of patients with both diseases having positive $\mathrm{RF}(P=0.027)$. Anti-CCP was not found to have a significant presence in co-existing RA and thyroid dysfunction, while CRP was elevated (3.21\%) without a statistical significance $(P=0.073)$. Whether these markers represent a significant relationship between the presence of clinical hypothyroidism and RA disease activity should be assessed further. The response of the levels of inflammatory markers to treatment of the thyroid disease should also be examined.

Given our findings, it appears that RA and autoimmune thyroid dysfunction are indeed linked. However, it is difficult to assess retrospectively which disorder predates the other, or if in fact there is such a temporal relationship.

It is be prudent to recommend screening patients with RA for thyroid dysfunction, perhaps more rigorously and frequently than in normal population. Symptoms of thyroid dysfunction should screened for routinely in patients with RA, and more frequent testing for thyroid disease should be undertaken if symptoms suggestive of dysfunction are detected. On the other hand, these symptoms should be systematically and scrupulously researched in the RA patients, all over their follow-up, given this high prevalence of comorbid thyroid dysfunction demonstrated by our study. For example, fatigue is a frequent symptom in patients with RA, and is also a symptom of hypothyroidism; therefore, screening for hypothyroidism - due to a further co-existing AITD- should be considered, especially if other causes of fatigue have been ruled out and pain controlled [19].

However, our study did not assess the effect of treating thyroid dysfunction on rheumatoid arthritis disease activity. Looking forward, examining the effect of treating thyroid dysfunction in patients with established RA on RA disease activity, symptomatology, and prognosis, as well as patient quality of life, is our next area of research. Screening for onset of thyroid dysfunction in established RA is another area of interest, as is establishing an idea of lag time between the onset of RA and AITD.

\section{CONCLUSION}

There is a high frequency of comorbid hypothyroidism in our RA patients, in Saudi Arabia, which suggests a high prevalence of Hashimoto's thyroiditis or other auto-immune thyroid dysfunctions, in these patients. Physicians should be aware of screening periodically RA patients for this comorbidity, especially when the specific symptoms may overlap between both diseases. Furthermore, the effect of treating thyroid dysfunctions in patients with established RA on RA disease activity could be an interesting area of research.

\section{AUTHORS' CONTRIBUTION}

Dr. Hala Mosli: Literature review, data analysis, preparation of manuscript, review of manuscript, submission.

Dr. Suzan Attar: Literature review, data collection, preparation of manuscript, review of manuscript.

\section{CONFLICT OF INTEREST}

The authors confirm that this article content has no conflict of interest.

\section{ACKNOWLEDGEMENTS}

Declared none.

\section{REFERENCES}

[1] Shiroky JB, Cohen M, Ballachey ML, Neville C. Thyroid dysfunction in rheumatoid arthritis: a controlled prospective survey. Ann Rheum Dis 1993; 52(6): 454-6.

[2] Roldán JC, Amaya-Amaya J, Castellanos-de la Hoz J, et al. Autoimmune thyroid disease in rheumatoid arthritis: a global perspective. Arthritis 2012.864907.

[3] Assal HS, Elsherbiny A, Alsayed A, Maaboud MA, Alshabrawi H, Abdel Rasheed E. Thyroid dysfunction in patients with systemic connective tissue disease. Maced J Med sci 2009; 2(3): 223-9. Doi:10.3889/MJMS. 1857-5773.2009.0059.

[4] El-Sherif WT, El-Gendi SS, Ashmawy MM, Ahmed HM, Salama MM. Throid disorders and autoantibodies in systemic lupus erythematosus and rheumatoid arthritis. Egypt J Immunol 2004; 11(2): 81-90. PMID:16734120.

[5] Mousa A A, Ghonem M, Hegazy A, El-Baiomy AA, El-Diasty A. Thyroid function and auto-antibodies in Egyptian patients with systemic lupus erythematosus and rheumatoid arthritis. Trends Med Res 2012; 7(1): 25 .

[6] Al-Awadhi AM ${ }^{1}$, Olusi S, Hasan EA, Abdullah A. Frequency of abnormal thyroid function tests in Kuwaiti Arabs with autoimmune diseases. Med Princ Pract 2008; 17(1): 61-5.

[7] Al-Dalaan A, Al Ballaa S, Bahabri S, Biyari T, Al Sukait M, Mousa M. The prevalence of rheumatoid arthritis in the Qassim region of Saudi Arabia. Ann Saudi Med 1998; 18(5): 396-7.

[8] Akbar DH, Ahmed MM, Al-Mughales J. Thyroid dysfunction and thyroid autoimmunity in Saudi type 2 diabetics. Acta Diabetol 2006; 43(1): 14-8.

[9] Neogi T, Aletaha D, Silman AJ, et al. The 2010 American College of Rheumatology/European League against rheumatism classification criteria for rheumatoid arthritis. Arthritis Rheum 2010; 62(9): 2582-91.

[10] Ngo ST, Steyn FJ, McCombe PA. Gender differences in autoimmune disease. Front Neuroendocrinol 2014; 35(3): 347-69. ISSN 0091-3022. http://dx.doi.org/10.1016/j.yfrne.2014.04.004.

[11] Jensen E, Blaabjerg O, Hansen PS, et al. Establishment of a serum thyroid stimulating hormone (TSH) reference interval in healthy adults. The importance of environmental factors, including thyroid antibodies. Clin Chem Lab Med 2004; 42(7): 824-32.

[12] Hamilton TE, Davis S, Onstad L, Kopecky KJ. Thyrotropin levels in a population with no clinical, autoantibody, or ultrasonographic evidence of thyroid disease: implications for the diagnosis of subclinical hypothyroidism. J Clin Endocrinol Metab 2008; 93(4): 1224-30.

[13] Tomer Y, Huber A. The etiology of autoimmune thyroid disease: a story of genes and environment. J Autoimmun 2009; 32(3-4): 23139.

[14] Tomer Y. Genetic susceptibility to autoimmune thyroid disease: past, present, and future. Thyroid 2010; 20(7): 715-25. 
[15] Barton A, Worthington J. Genetic susceptibility to rheumatoid arthritis: an emerging picture. Arthrit Care Res 2009; 61(10): 14416.

[16] Roberts CG, Ladenson PW. Hypothyroidism. Lancet 2004; 363(9411): 793-803

[17] Park EA, Jerden DC, Bahouth SW. Regulation of phosphoenolpyruvate carboxykinase gene transcription by thyroid hormone involves two distinct binding sites in the promoter. Biochem $\mathbf{J}$ 1995; 309(Pt 3): 913-9.
[18] Raterman HG, Van Halm VP, Voskuyl AE, Simsek S, Dijkmans BA, Nurmohamed MT. Increased prevalence of antithyroid antibodies in rheumatoid arthritis (RA) patients with a low prevalence of hormonal alterations. Autoimmunity 2008; 41(5): 337.

[19] Pollard LC, Choy EH, Gonzalez J, Khoshaba B, Scott DL. Fatigue in rheumatoid arthritis reflects pain, not disease activity. Rheumatology 2006; 45(7): 885-9. doi: 10.1093/rheumatology /kel021, First published online: January 31, 2006.

(C) Mosli and Attar; Licensee Bentham Open.

This is an open access article licensed under the terms of the Creative Commons Attribution Non-Commercial License (http://creativecommons.org/licenses/bync/3.0/) which permits unrestricted, non-commercial use, distribution and reproduction in any medium, provided the work is properly cited. 